http://dx.doi.org/10.35381/e.k.v3i5.835

\title{
La Realidad Virtual como herramienta de innovación educativa
}

\section{Virtual Reality as a tool for educational innovation}

\author{
Josefa Katiuska Toala-Palma \\ josefatoala@yahoo.com \\ Pontificia Universidad Católica del Ecuador, Extensión Manabí, Portoviejo \\ Ecuador \\ https://orcid.org/0000-0002-5298-6321 \\ Jéssica Lourdes Arteaga-Mera \\ jessicaarteaga2010@hotmail.com \\ Pontificia Universidad Católica del Ecuador, Extensión Manabí, Portoviejo \\ Ecuador \\ https://orcid.org/0000-0002-0587-0166 \\ Juana Maricela Quintana-Loor \\ quintanamaricela@hotmail.com \\ Pontificia Universidad Católica del Ecuador, Extensión Manabí, Portoviejo \\ Ecuador \\ https://orcid.org/0000-0002-4396-2854 \\ María Isabel Santana-Vergara \\ ambarabel@hotmail.com \\ Pontificia Universidad Católica del Ecuador, Extensión Manabí, Portoviejo \\ Ecuador \\ https://orcid.org/0000-0001-8491-4165
}

Recepción: 19 abril 2020

Revisado: 26 de mayo 2020

Aprobación: 22 junio 2020

Publicación: 26 de junio 2020 


\title{
RESUMEN
}

La investigación de revisión tuvo como objetivo analizar la realidad virtual como herramienta de innovación educativa. Metodológicamente se desarrolló desde una tipología descriptiva - documental con diseño bibliográfico no experimental, aplicándose la técnica de análisis de contenido para la selección e interpretación de la información recopilada de revistas ubicadas en bases de datos como Scopus, WOS, Scielo, Latindex 2.0. El conocimiento escolar puede ser profundizado en la medida que se apoya en recursos tecnológicos, posibilitando un ejercicio pedagógico en consonancia con las tendencias actuales La realidad virtual en escenarios educativos, donde ha sida implementada, desencadena efectos positivos en el rendimiento académico de los estudiantes, situación que conlleva a pesar en la necesidad de su implementación en el sistema educativo ecuatoriano, para lo cual, se requiere de inversión y adaptación de los espacios académicos para configurar un aprendizaje significativo desde la realidad virtual.

Descriptores: Informática educativa; política educacional; programa informático didáctico; tecnología educacional. (Palabras tomadas del Tesauro UNESCO).

\begin{abstract}
The review research aimed to analyze virtual reality as an educational innovation tool. Methodologically, it was developed from a descriptive-documentary typology with nonexperimental bibliographic design, applying the content analysis technique for the selection and interpretation of the information collected from journals located in databases such as Scopus, WOS, Scielo, and Latindex 2.0. School knowledge can be deepened to the extent that it relies on technological resources, enabling a pedagogical exercise in line with current trends. Virtual reality has triggered positive effects on the academic performance of students in the educational settings where it has been implemented, a situation that leads to thinking about the need of its implementation in the Ecuadorian educational system, for which it requires investment and adaptation of academic spaces to configure meaningful learning from virtual reality.
\end{abstract}

Descriptors: Computer uses in education; educational policy; educational software; educational technology. (Words taken from the UNESCO Thesaurus). 


\section{INTRODUCCIÓN}

Existen varias definiciones de lo que significa Realidad Virtual, la cual se denominará RV de aquí en adelante, una de ellas la describe como la interface hombre-máquina (humanmachine), la cual le da la oportunidad a la persona de ahondarse en una simulación gráfica de 3 dimensiones (3D) la cual genera una computadora que permite navegar interactuando en ella en tiempo real (Pérez-Martínez, 2011). La RV es una simulación que genera una computadora en 3D, en el cual el avatar o usuario puede ver y manipular el contenido, una de sus características es que genera una respuesta multisensorial e interactiva lo cual permite una sensación inmediata a lo real (León-Guerra, 2012).

La información multisensorial que proporciona la RV es sonido, visión, el tacto, con la intención que el usuario pueda sentir que está en el lugar seleccionado, hasta hace poco tiempo tanto el software como hardware necesario para la RV era muy costoso, en este momento es más fácil por cuanto los precios de las computadoras o dispositivos se han visto reducidos logrando que muchas personas e instituciones educativas tengan la posibilidad de explorar en el mundo de la RV desde sus dispositivos personales.

Según las fuentes consultadas sobre $R V$ coinciden en lo que significa sumergirse en el mundo 3D, de lo que se observa y se vive al sentir que los objetos salen de la pantalla y con la sensación de acercarse a la persona, estas experiencias generan en los estudiantes un conocimiento, además permite comprender elementos abstractos y fenómenos complejos que de otra manera se comprenderían en menor porcentaje, la RV le permite experimentar con el mundo real. Existen dos tipos de realidad virtual, que depende del hardware que se utilice:

- RV de escritorio: La utiliza una persona o un grupo pequeño de personas, el ambiente virtual es proyectado a través de una computadora y las personas interactúan utilizando puede ser el mouse para navegar en 3D o puede ser los guantes de datos. 
Josefa Katiuska Toala-Palma; Jéssica Lourdes Arteaga-Mera; Juana Maricela Quintana-Loor; María Isabel Santana-Vergara

- RV de inmersión: La persona utiliza un casco de RV o unos lentes, sensores de movimiento y guantes de datos esto permite al usuario logar un alto porcentaje de inmersión en la realidad. (Urquiza-Mendoza, Auria-Burgos, \& Daza-Suarez, 2016).

Los mundos virtuales pueden estar diseñados para una sola persona llamado en este caso usuario o para muchos usuarios simultáneos. Cuando ese mundo virtual soporta varios usuarios da lugar a lo que se le llama comunidad virtual. Los usuarios que forman parte de esta comunidad virtual tienen un nivel cognitivo elevado en el mundo artificial, en ese contexto simulado estos usuarios pueden comunicarse con otros usuarios, en el plano del trabajo colaborativo, enseñanza y aprendizaje, esta comunidad de aprendizaje cambia el rol del profesor y del estudiante ya que se convierten en usuarios instructores y aprendices el aprendizaje en el entorno de la RV podría ser más significativo (Jimenez, Villalobos-Abarca \& Luna-Ramírez, 2000).

Se considera pertinente conocer el concepto o la concepción de educación ideal para contrastarla con la real y de esta manera tomar conciencia sobre las cosas que es necesario modificar. En proceso de educación hace lo posible para encaminar, guiar, orientar, potenciar, desarrollar o perfeccionar las facultades intelectuales, morales y físicas del estudiante a través del proceso de la enseñanza que en muchos casos puede ser experiencial orientados por el docente, guía o tutor facultado para este fin (ZambranoFerre, 2005).

La incorporación de la RV a las aulas como innovación de la educación del Siglo XXI supone un salto cualitativo importante en el proceso de aprendizaje en las distintas áreas de conocimiento, de manera especial en aquellas que es difícil visualizar los procesos que se han estudiado. Esta tecnología facilita al docente la explicación de procesos complejos (Urquiza-Mendoza, Auria-Burgos, \& Daza-Suarez, 2016).

La RV se hace cada vez más popular en los Instituciones educativas por su amplia variedad de aplicaciones, por ejemplo el museo virtual que ayuda a fortalecer el estudio 
Josefa Katiuska Toala-Palma; Jéssica Lourdes Arteaga-Mera; Juana Maricela Quintana-Loor; María Isabel Santana-Vergara

de historia, el patrimonio de determinados países y el arte, así mismo favorece que los alumnos se involucren en hechos históricos o de ficción enriqueciendo y haciendo más significativos sus conocimientos, también pueden hacer estudios de las baterías que entran en el cuerpo de los humanos y ocasionan enfermedades, esta tecnología utilizada en la Educación del Siglo XXI permita que lo abstracto e intangible pase a ser concreto y manipulable. La RV tiene ventajas en la Educación, incluida la entrega de información a través de canales, la inclusión valorando los distintos estilos de aprendizaje y sobre todo el aprendizaje experiencial y significativo (Flores-Cruz, Camarena-Gallardo \& Avalos-Villarreal, 2014).

La innovación, los avances, la investigación y las nuevas tecnologías han generado cambios y necesidades de aprendizajes. La sociedad ha planeado exigencias en la educación que requiere modelos de enseñanza que se adapten al tiempo y al espacio. Las TIC ofrecen variedad de posibilidades para la educación específicas en función a la necesidad de los estudiantes que se quieren formar y en función al contenido que se quiere impartir, por lo tanto son medio que permite implementar nuevos modelos pedagógicos para la enseñanza y el aprendizaje (Olivar \& Daza, 2007).

De ese modo, en virtud de lo novedoso que representa la realidad virtual para la educación, sobre todo por la inversión tecnológica que debe realizarse, la presente investigación se basa en una revisión documental de investigaciones publicadas en revistas arbitradas con la finalidad de conocer los aportes y tendencias de la RV al campo educativo, por lo cual se ha trabajado en base a una tipología descriptiva - documental, desde la perspectiva de (Hernández, Fernández \& Baptista (2014), aplicándose el análisis de contenido como técnica para el análisis de la información, lo cual ha permitido plantearse el siguiente objetivo analizar la realidad virtual como herramienta de innovación educativa. 
Josefa Katiuska Toala-Palma; Jéssica Lourdes Arteaga-Mera; Juana Maricela Quintana-Loor; María Isabel Santana-Vergara

\section{La Realidad Virtual como herramienta de innovación educativa}

El campo de la realidad virtual se torna diverso en su utilidad, siendo pionero para el campo de los video juegos y publicidad, luego se ha implementado en la industria médica, progresivamente ha sido asumido con fines educativos, (Ruiz-Davis \& Polo-Serrano, 2012), contribuyen al indicar que la realidad virtual aumentada, tiene la capacidad de entretener y generar aprendizaje en la persona, dentro del horario a sus actividades escolares.

La realidad aumentada parte como una propuesta de entretenimiento que ha sido adapta progresivamente a usos diversos en función de las necesidades e intereses de las empresas, lo cual se configura en la medida que la tecnología diseña nuevos modelos de dispositivos móviles en sus diversas presentaciones, consolas de juego, tablet, los cuales se constituyen en atractivos para los jóvenes, situación que es asumida por la educación al incorporar en primer lugar las TIC, para progresivamente involucrar la realidad aumentada como estrategia pedagógica atractiva para los estudiantes en virtud de promover un mejor aprendizaje.

Por otro lado, (Prendes-Espinosa, 2015), comentan que la realidad aumentada en el sector educativa no pasará desapercibida, esto conlleva a la idea de que la educación de la próxima década estará impregnada por la realidad aumentada, más aun cuando ya existen las aulas 2.0, aunado a la evolución tecnológica propia de un mundo globalizado, lo cual no puede ser indiferente para quienes diseñan los programas curriculares, quienes deberán tener presente la realidad aumentada como parte del mismo. En complemento, Del-Cerro-Velázquez \& Morales-Méndez (2017), destacan que:

La implementación de una aplicación didáctica, basada en recursos TIC, para el alumnado de 3ำ de la ESO en la materia Tecnologías ha sido una experiencia enriquecedora y motivadora, que nos ha permitido comprobar la actitud positiva y tremendamente receptiva del alumnado ante este tipo de experiencias (p. 12). 
La motivación al aprendizaje es una tarea que padres, representantes y docentes deben afrontar para estimular asertivamente a los estudiantes al logro de un aprendizaje significativo tal como indican, (Vallejo-Valdivieso et al., 2019), siendo la realidad aumentada una alternativa viable por cuanto el mundo tecnológico se percibe motivador para los estudiantes, lo cual puede implementarse con fines educativos, las organizaciones educativas deben invertir en dispositivos tecno0lógicos, así como programas que permitan generar aprendizajes desde la realidad aumentada, las universidades están llamadas a diseñar y promover la realidad aumentada con fines educativos y didácticos. En este sentido, (Cabero-Almenara, Barroso-Osuna \& LlorenteCejudo (2016), señalan que:

La RA es una tecnología que se está presentando como de verdadera utilidad y con diferentes posibilidades para facilitar el aprendizaje por parte de los estudiantes en diferentes áreas curriculares, pero sobre la que se debe reconocer que se están efectuando más análisis tecnológicos que investigaciones sobre su aplicación en el terreno educativo.

La realidad aumentada puede ser implementada transversalmente en el currículo, siendo posible ser implementada en diversas materias o en proyectos de aprendizaje, los estudiantes podrán diseñar estrategias que le permitan concatenar la realidad virtual con los temas curriculares, posibilitándose la generación de aprendizajes en consonancia con las necesidades e intereses del estudiante. Es así que la realidad aumentada en conjunto con dispositivos tecnológicos se constituye en parte de la vida cotidiana de las nuevas generaciones, siendo ineludible por parte de las autoridades educativas no involucrar las TIC, realidad aumentada, así como otras tendencias tecnológicas e informáticas al campo educativo, aunado cuando se transita al paradigma de aprendizaje centrado en el estudiante, (Maya, Aldana-Zavala \& Isea-Argüelles, 2019), argumentan que tal medida concibe una educación de calidad e inclusiva. 
Desde la perspectiva planteada, la realidad aumentada puede ser implementada en diversos escenarios, lo cual puede aprovecharse con fines educativos, posibilitando el aprendizaje desde contextos no convencionales, siendo posible la generación educativa formal y no formal, (Del-Cerro-Velázquez \& Morales-Méndez, 2017), comentan la necesidad de introducir en la educación, la realidad aumentada, mediante el uso de teléfonos inteligentes, los cuales permiten el manejo de aplicaciones que pueden ser usadas con fines educativos, siendo así motivador para los estudiantes realizar un abordaje de actividades académicas por medio de dispositivos móviles.

Por otro lado, Villalustre-Martínez \& Del-Moral-Pérez (2018), destacan "la necesidad de introducir estas tecnologías en la formación de los futuros maestros para potenciar su integración didáctica en las aulas más allá de uso instrumental de una manera inclusiva y dinámica" (p. 25), esto es necesario por cuanto los maestros, docentes, al no estar familiarizados con las nuevas tecnologías, podrían asumir una postura reactiva al uso pedagógico de las mismas, siendo indispensable la generación de un cambio paradigmático en la educación en donde se incluya la realidad virtual como elemento innovador del aprendizaje, tal como indican (Lattá-Arias, 2019), (Urueta-Vélez, 2019), (Gómez, 2017). En complemento, Maquilón-Sánchez, Mirete-Ruiz \& Avilés-Olmos (2017), destacan las multiples variantes de la realidad virtual en su adaptación al ambiente de aprendizaje.

La realidad virtual permite generar un aprendizaje significativo y social, desde el aprovechamiento de los conocimientos previos de los estudiantes, así como la generación de competencias, siendo esto en concordancia con las nuevas tendencias curriculares donde se promueve la formación transversal y bioética del estudiante, mediante el proyecto de vida como estrategia generadora de un aprendizaje motivador a lo largo de la vida, construyéndose progresivamente una 
educación inclusiva y de calidad.

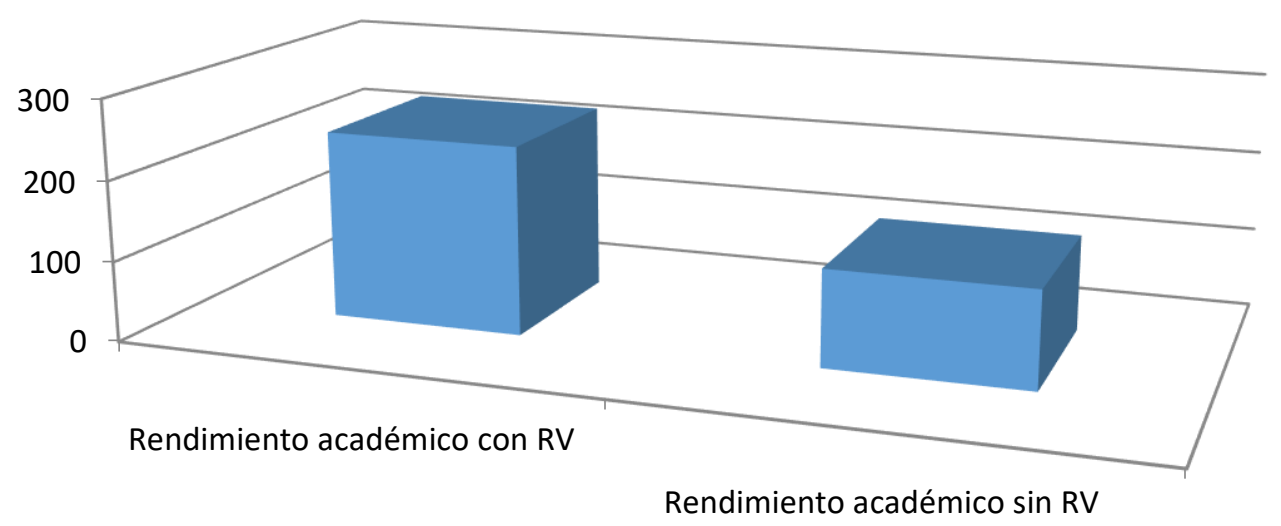

Figura 1. Diferencia de rendimiento académico. Fuente: Kraft, et al. 2018.

La diferencia entre los estudiantes en quienes se han implementado la realidad virtual como estrategia de aprendizaje, ha recopilado un mayor rendimiento académico que quienes no abordaron la realidad aumentada, lo cual a juicio de (Donnelly, et al., 2014), proyecta la importancia de este enfoque educativo en virtud de incentivar la motivación del estudiante a lograr un mejor aprendizaje (Carvalho-Beluce, \&, Luciane-de-Oliveira, 2015), así mismo, (Hsieh \& Yeehsieh, 2004), destacan que el conocimiento escolar puede ser profundizado en la medida que se apoya en recursos tecnológicos, posibilitando un ejercicio pedagógico en consonancia con las tendencias actuales (Chernikova, et al., 2020), lo cual transciende a estudiantes universitarios, quienes por medio de video juegos y simulación, han alcanzado un mayor aprendizaje en ingeniería (Bodnar, et al., 2016), lo cual proyecta la aplicación de la realidad educativa en los diversos niveles del sistema educativo, teniendo en cuenta que la realidad virtual permite 
Josefa Katiuska Toala-Palma; Jéssica Lourdes Arteaga-Mera; Juana Maricela Quintana-Loor; María Isabel Santana-Vergara

generar un pensamiento espacial en los estudiantes (Hod \& Twersky, 2020), asímismo (Danish, et al., 2020), indican que además contribuye a la generación de un aprendizaje colaborativo.

Por otro lado, (De-la-Torre-Cantero, et al., 2013), comentan que los estudiantes valoran el uso de la realidad virtual por medio de tabletas digitales en preferencia a otros dispositivos tecnológicos, (Cantón-Enríquez, et al., 2018), contribuye (Soto-Triana \& Gómez-Villamizar, 2018), comentan que la realidad virtual ha brindado buenos resultados en la psicología clínica para el control de la ansiedad, situación que puede ser focalizada a la educación, generándose estrategias de abordaje donde se contribuya en fomentar un mejor aprendizaje en los estudiantes con ansiedad, traumas, miedos, estrés, siendo apoyada por la investigación de (Campos-Soto, et al., 2020), quienes aseguran que la calidad educativa mejora mediante la implementación de la realidad virtual.

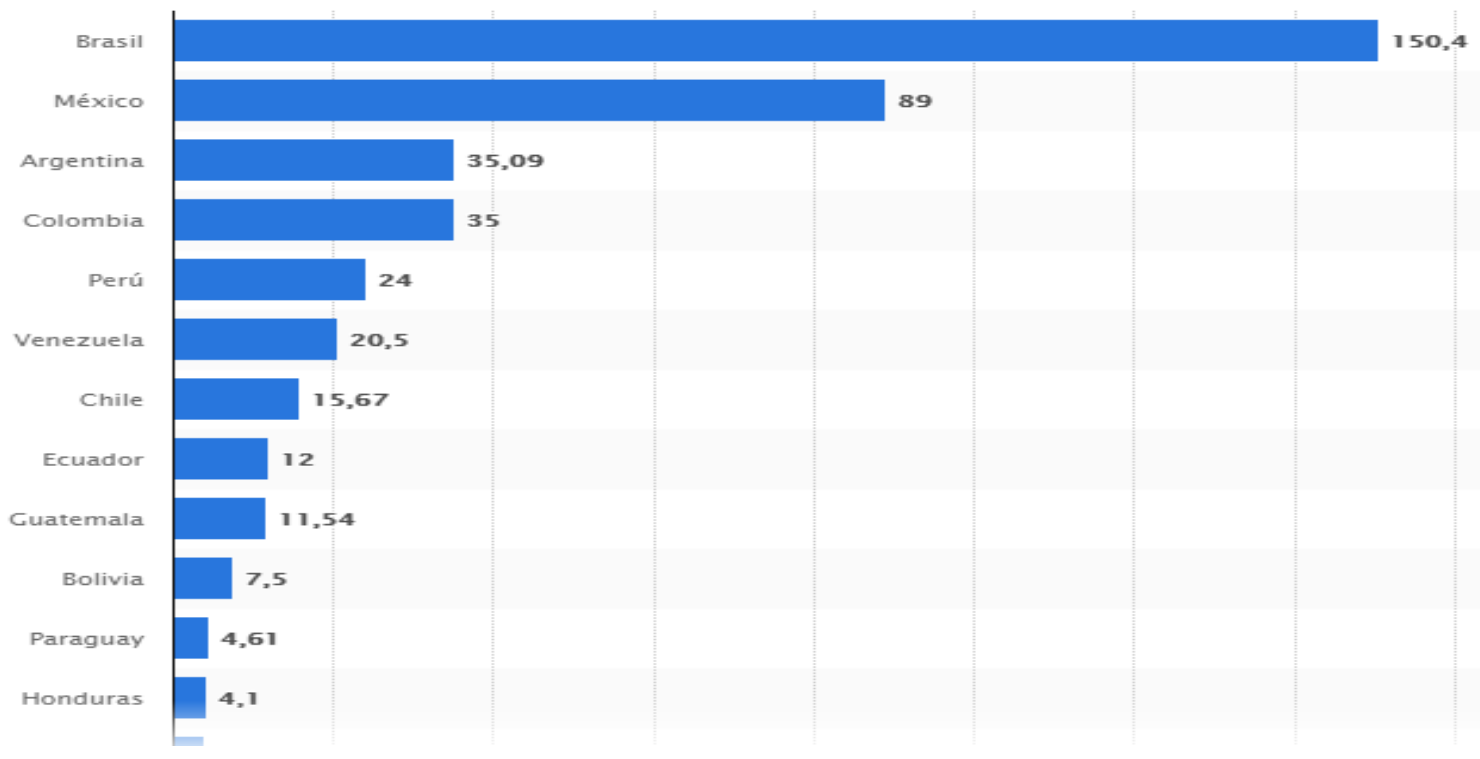

Figura 2. Usuarios de internet en Latinoamérica 2020. Fuente. Burgueño-Salas, 2020. 
Una de las limitaciones en Latinoamérica para el uso efectivo de la realidad virtual con fines educativos, es la brecha existente en el acceso al internet, así como el equipamiento tecnológico necesario para que el estudiante pueda desarrollar esta modalidad de aprendizaje, sobre todo en países donde el ingreso económico mensual de las familias no permite contar con el poder adquisitivo necesario para equipar por ejemplo a dos estudiantes, siendo necesaria la implementación de políticas públicas en razón de contar con planes de financiamiento para el sostenimiento de la realidad virtual, sin embargo, esta debilidad o limitante puede constituirse en una oportunidad, si se mira desde la óptica de la educación con fines de emprendimiento, lo cual puede promover en el estudiante la capacidad de crear nuevos sistemas informáticos que podrían ser patentados con fines de reponer lo invertido.

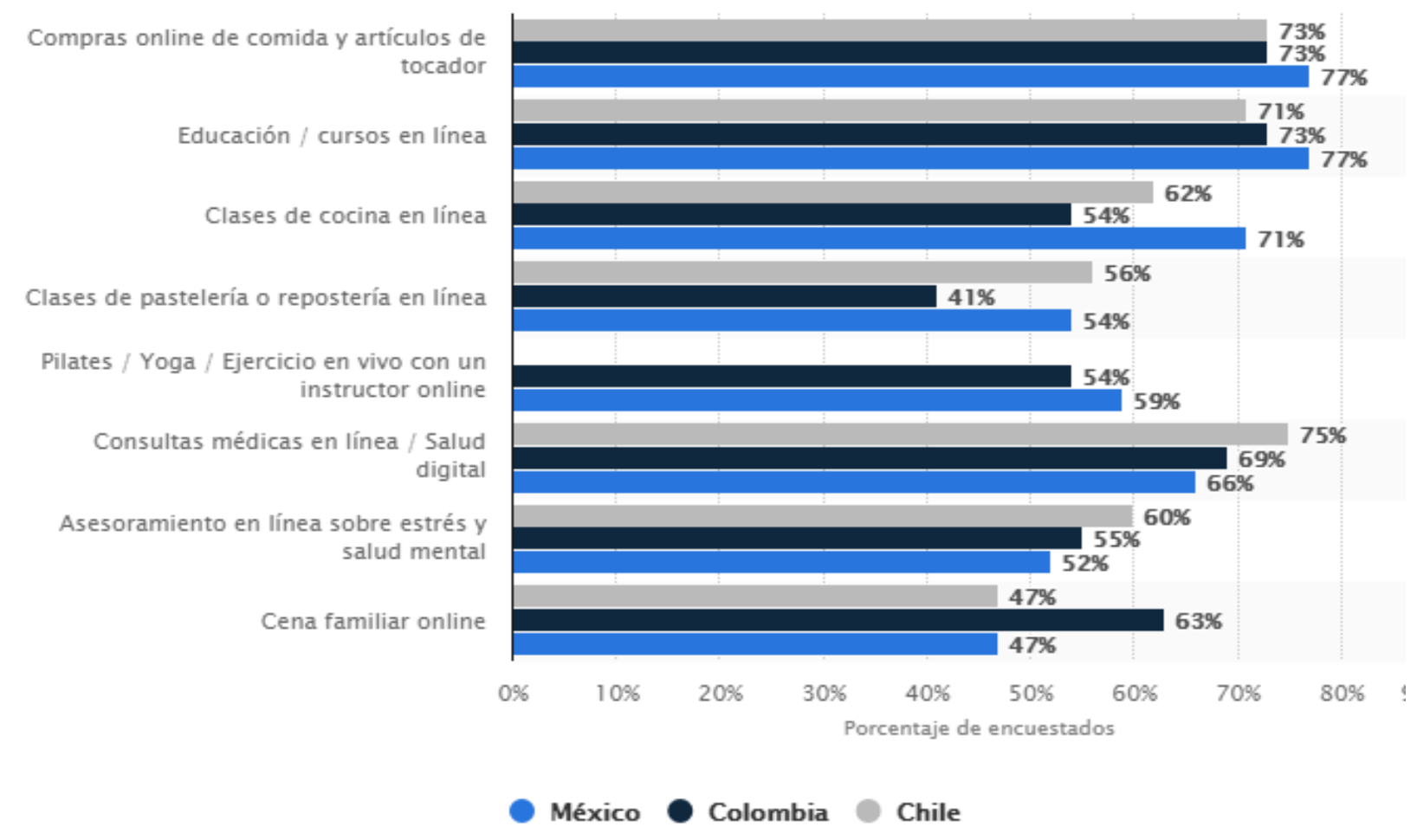

Figura 3. Actividades online desde pandemia COVID-19. Fuente. Mendoza, 2020. 
La figura 3, evidencia las actividades generadas en línea durante la pandemia, lo cual visualiza dos eventos: 1 . El cambio inminente hacia lo virtual de la gran mayoría de rubros comerciales, 2. No adaptarse a tal realidad, implica el riesgo de perecer en el mercado laboral; lo cual incluye lo educativo, siendo que se debe educar con fines de abordar la virtualidad en el presente y futuro por parte de los estudiantes, por cuanto es en ese segmento económico donde se concentrarán la mayor cantidad de empleo en los próximos años, siendo un rol de la educación, formar para la vida, lo cual refuerza la idea anterior de emprender desde la educación.

\section{CONCLUSIÓN}

La realidad virtual en escenarios educativos, donde haya sido puesta en práctica, ha desencadenado efectos positivos en el rendimiento académico de los estudiantes; situación que conlleva a pensar en la necesidad de su implementación en el sistema educativo ecuatoriano, para lo cual, se requiere de inversión y adaptación de los espacios académicos para configurar un aprendizaje significativo desde la realidad virtual. Asímismo, puede contribuir en promover en los estudiantes las inteligencias múltiples por cuanto se emplean los diversos procesos lógicos - matemáticos, espacial y artístico, para diseñar, programar, aplicar, la realidad virtual con fines pedagógicos, lo cual podría además, proyectar la generación de nuevas habilidades y competencias, siendo favorecedor de una educación constructivista con enfoque tecnológico.

\section{FINANCIAMIENTO}

No monetario 
Josefa Katiuska Toala-Palma; Jéssica Lourdes Arteaga-Mera; Juana Maricela Quintana-Loor; María Isabel Santana-Vergara

\section{AGRADECIMIENTOS}

A la Pontificia Universidad Católica del Ecuador, Sede Manabí, Campus Chone y al Doctor Patricio Alfredo Vallejo-Valdivieso por estimular e impulsar esta inquietud investigativa desde la praxis educativa.

\section{REFERENCIAS}

Bodnar, C.A., Anastasio, D., Enszer, J.A. \& Burkey, D.D. (2016), Engineers at Play: Games as Teaching Tools for Undergraduate Engineering Students. [Ingenieros en juego: juegos como herramientas de enseñanza para estudiantes de ingeniería de pregrado]. J. Eng. Educ., 105: 147-200. https://doi.org/10.1002/jee.20106

Burgueño-Salas, S. (2020). América Latina: usuarios de internet por país 2020. Statista. Recuperado de https://n9.cl/7fp9

Carvalho-Beluce, A, \&, Luciane-de-Oliveira, K. (2015). Motivación de los estudiantes para aprender en entornos virtuales de aprendizaje. [Students' Motivation for Learning in Virtual Learning Environments1]. Paidéia (Ribeirão Preto). 25 (60), 105113. https://doi.org/10.1590/1982-43272560201513

Cabero-Almenara, J., Barroso-Osuna, J. \& Llorente-Cejudo, M. (2016). Technology acceptance model \& realidad aumentada: estudio en desarrollo. [Technology acceptance model \& augmented reality: study in progress]. Revista Lasallista de Investigación, Vol. 13 No. 2, 18-26. https://doi.org/10.22507/rli.v13n2a2

Cantón-Enríquez, D., Arellano-Pimentel, J., Hernández-López, M., \& Nieva-García, O. (2017). Uso didáctico de la realidad virtual inmersiva con interacción natural de usuario enfocada a la inspección de aerogeneradores. [Didactic use of immersive virtual reality with $\mathrm{NUI}$ focused on the inspection of wind turbines]. Apertura (Guadalajara Jal.), 9(2), 8-23. http://dx.doi.org/10.32870/Ap.v9n2.1049

Campos-Soto, M., Ramos-Nava-Parejo, M., \& Moreno-Guerrero, A. (2020). Realidad virtual y motivación en el contexto educativo: Estudio bibliométrico de los últimos veinte años de Scopus. Revista de Educación Alteridad, 15(1), 47-60. https://doi.org/10.17163/alt.v15n1.2020.04 
Josefa Katiuska Toala-Palma; Jéssica Lourdes Arteaga-Mera; Juana Maricela Quintana-Loor; María Isabel Santana-Vergara

Chernikova, O., Heitzmann, N., Stadler, M., Holzberger, D., Seidel, T., \& Fischer, F. (2020). Simulation-Based Learning in Higher Education: A Meta-Analysis. [Aprendizaje basado en simulación en la educación superior: un metanálisis]. Review of Educational Research. https://doi.org/10.3102/0034654320933544

Danish, J.A., Enyedy, N., Saleh, A. \& Humburg, M. (2020). Learning in embodied activity framework: a sociocultural framework for embodied cognition. Intern. J. Comput.Support. Collab. Learn 15, 49-87 (2020). https://doi.org/10.1007/s11412-020$\underline{09317-3}$

Del-Cerro-Velázquez, F. \& Morales-Méndez, G. (2017). Realidad Aumentada como herramienta de mejora de la inteligencia espacial en estudiantes de educación secundaria. [Augmented Reality as a tool for improving spatial intelligence in secondary education students]. RED. Revista de Educación a Distancia. Núm. 54. http://dx.doi.org/10.6018/red/54/5

De-la-Torre-Cantero, J, \& Martin-Dorta, N, \& Saorín-Pérez, J, \& Carbonell-Carrera, C, \& Contero-González, M. (2013). Entorno de aprendizaje ubicuo con realidad aumentada y tabletas para estimular la comprensión del espacio tridimensional. [Ubiquitous learning environment with augmented reality and tablets to stimulate understanding of three-dimensional space]. RED. Revista de Educación a Distancia, (37), 1-17. Recuperado de https://n9.cl/fzhre

Donnelly, D. F., Linn, M. C., \& Ludvigsen, S. (2014). Impacts and Characteristics of Computer-Based Science Inquiry Learning Environments for Precollege Students. [Impactos y características de los entornos de aprendizaje de la investigación científica basada en computadora para estudiantes de precolegio]. Review of Educational Research, 84(4), 572-608. https://doi.org/10.3102/0034654314546954

Flores-Cruz, J., Camarena-Gallardo, P., \& Avalos-Villarreal, E. (2014). La realidad virtual, una tecnología innovadora aplicable al proceso de enseñanza de los estudiantes de ingeniería. [Virtual reality, an innovative technology applicable to the teaching process of engineering students]. Apertura, 6 (2), 1-10. Recuperado de https://n9.cl/ruw40

Gómez. J. (2017). Experiencia en el diseño de instrumento para la virtualidad. [Experience in instrument design for virtuality]. Revista Arbitrada Interdisciplinaria Koinonía, 2(3), 65-79. Recuperado de https://n9.cl/061f 
Josefa Katiuska Toala-Palma; Jéssica Lourdes Arteaga-Mera; Juana Maricela Quintana-Loor; María Isabel Santana-Vergara

Hsieh, S.J. \& Yeehsieh, P. (2004), Integrated Virtual Learning System for Programmable Logic Controller. [Sistema de aprendizaje virtual integrado para controlador lógico programable]. Journal of Engineering Education, 93: 169-178. https://doi.org/10.1002/j.2168-9830.2004.tb00801.x

Hernández, R. Fernández, C., \& Baptista, P. (2014). Metodología de la investigación. [Investigation methodology]. México, Mc Graw Hill Hispanoamericana. Hill Internacional.

Hod, Y, \& Twersky, D. (2020). Distributed spatial Sensemaking on the augmented reality sandbox. Intern. J. Comput.-Support. Collab. Learn 15, 115-141. https://doi.org/10.1007/s11412-020-09315-5

Jimenez , A., Villalobos-Abarca, M., \& Luna-Ramírez, E. (2000). Cuándo y Cómo usar la Realidad Virtual en la Enseñanza. [When and How to use Virtual Reality in Teaching]. Iberoamericana de Informática Educativa (16), 26-36. Recuperado de https://n9.cl/htsrk

Kraft, M. A., Blazar, D., \& Hogan, D. (2018). The Effect of Teacher Coaching on Instruction and Achievement: A Meta-Analysis of the Causal Evidence. [El efecto del coaching docente sobre la instrucción y el rendimiento: un metaanálisis de la evidencia causal]. Review of Educational Research, 88(4), 547-588. https://doi.org/10.3102/0034654318759268

Lattá-Arias, C. (2019). Uso de las TIC para proyectos productivos en las instituciones educativas del Municipio Zona Bananera. Magdalena. Colombia. [Use of ICT for productive projects in educational institutions of the Municipality of the Banana Zone. Cupcake Colombia]. Revista Arbitrada Interdisciplinaria Koinonía, 4(7), 233246. http://dx.doi.org/10.35381/r.k.v4i7.202

León-Guerra, R. (2012). Aplicación de la realidad virtual no inmensiva para Ingerieros Agrícolas. [Non-immersive virtual reality application for Agricultural Engineers]. Ciencias Técnicas Agropecuarias, 21(1), 69-71. Recuperado de https://n9.cl/1q3no

Maquilón-Sánchez, J.J., Mirete-Ruiz, A.B. \& Avilés-Olmos, M. (2017). La Realidad Aumentada (RA).Recursos y propuestas para la innovación educativa. [Augmented Reality (AR). Resources and proposals for educational innovation]. Revista Electrónica Interuniversitaria de Formación del Profesorado, 20(2), 183-203. https://doi.org/10.6018/reifop/20.2.290971 
Josefa Katiuska Toala-Palma; Jéssica Lourdes Arteaga-Mera; Juana Maricela Quintana-Loor; María Isabel Santana-Vergara

Mendoza, J. (2020). América Latina: tendencias de consumo online desde la pandemia de COVID-19. Recuperado de https://n9.cl/i3ow

Maya, E., Aldana-Zavala, J., \& Isea-Argüelles, J. (2019). Liderazgo Directivo y Educación de Calidad. [Management Leadership and Quality Education]. CIENCIAMATRIA, 5(9), 114 - 129. https://doi.org/10.35381/cm.v5i9.102

Olivar, A., \& Daza, A. (2007). Las tecnologías de la información y comunicación (TIC) y su impacto en la educación del siglo XXI. [Information and communication technologies (ICT) and their impact on education in the 21st century]. NEGOTIUM , 3(7), 21-46. Recuperado de https://n9.cl/2psi

Pérez-Matínez, F. (2011). Presente y Futuro de la Tecnología de la Realidad Virtual. Creatividad y Sociedad (18), 1-39. Recuperado de https://n9.cl/uzrd

Prendes-Espinosa, C. (2015). Realidad aumentada y educación: análisis de experiencias prácticas. [augmented realityand education: analysis of practical experiencies]. Pixel-Bit. Revista de Medios y Educación, (46), 187-203. Recuperado de https://n9.cl/348I

Ruiz-Davis, S., \& Polo-Serrano, D. (2012). La realidad aumentada como nuevo concepto de la publicidad en línea a través de los smartphones. [Augmented reality as a new concept of online advertising through smartphones]. Razón y Palabra, (80). Recuperado a partir de https://n9.cl/h1m1b

Soto-Triana, J. S., \& Gómez-Villamizar, C. (2018). Avances en realidad virtual e intervenciones en psicología [Virtual Reality Progress and Interventions in Clinical Psychology]. clínica. Tesis Psicológica, 13(1), 1-15. https://doi.org/10.37511/tesis.v13n1a5

Urueta-Vélez, L. (2019). Estrategias de enseñanza y el uso de las tecnologías de información y comunicación en las instituciones educativas departamentales en el Municipio Zona Bananera - Colombia. [Teaching strategies and the use of information and communication technologies in departmental educational institutions in the Zona Bananera Municipality - Colombia]. Revista Arbitrada Interdisciplinaria Koinonía, 4(7), 185-201. http://dx.doi.org/10.35381/r.k.v4i7.200

Urquiza-Mendoza, L., Auria-Burgos, B., \& Daza-Suarez, S. (2016). Uso de la realidad virtual, en la educación del futuro en centros educativos del Ecuador. [Use of 
Josefa Katiuska Toala-Palma; Jéssica Lourdes Arteaga-Mera; Juana Maricela Quintana-Loor; María Isabel Santana-Vergara

virtual reality in the education of the future in educational centers in Ecuador]. Ciencia e Investigación, 1(4), 26-30. Recuperado de https://n9.cl/a7ii

Vallejo-Valdivieso, P., Zambrano-Pincay, G., Vallejo-Pilligua, P., \& Bravo-Cedeño, G. (2019). Estructuras mentales en la construcción de aprendizaje significativo. [Mental structures in the construction of meaningful learning]. CIENCIAMATRIA, 5(8), 228-241. https://doi.org/10.35381/cm.v5i8.257

Villalustre-Martínez, L. \& Del-Moral-Pérez, E. (2018). Geolocalización y realidad aumentada para un aprendizaje ubicuo en la formación inicial del profesorado. [Geolocation and augmented reality for ubiquitous learning in initial teacher training]. @tic revista d 'innovació educativa. 21(18), 19-27. https://doi.org/10.7203/attic.21.12633

Zambrano-Ferre, A. (2005). Aspectos Éticos del uso de la Realidad Virtual en la Enseñanza de la Anatomía Humana. [Ethical Aspects of the Use of Virtual Reality in the Teaching of Human Anatomy]. Fermentum, 15(44), 426-438. Recuperado de https://n9.cl/hn20 